\title{
Non-Markovian Forward-Backward Stochastic Differential Equations with Discontinuous Coefficients
}

\author{
Yin Hong \\ Department of Mathematics, State University of New York, Brockport, NY, USA \\ Email: hyin@brockport.edu
}

How to cite this paper: Hong, Y. (2020) Non-Markovian Forward-Backward Stochastic Differential Equations with Discontinuous Coefficients. Applied Mathematics, 11, 328-343.

https://doi.org/10.4236/am.2020.114024

Received: March 10, 2020

Accepted: April 20, 2020

Published: April 23, 2020

Copyright (c) 2020 by author(s) and Scientific Research Publishing Inc. This work is licensed under the Creative Commons Attribution International License (CC BY 4.0).

http://creativecommons.org/licenses/by/4.0/

\begin{abstract}
In this paper, the existence and uniqueness of a weak solution in the sense of [1] and [2] has been shown for a class of fully coupled forward-backward SDE (FBSDE) such that the forward drift coefficient is allowed to be discontinuous with respect to the backward component of the solution. The novelty of this paper lies on the fact that the FBSDE is non-Markovian, i.e., the coefficients of the FBSDEs are allowed to be random. This type of FBSDEs is inspired by the regime shift model, where the short term interest rate switches between regimes according to the rate level. As a consequence, the discontinuity of the system becomes inevitable, making it violate the usual assumptions of most existing results for FBSDEs. We show the weak well-posedness of the FBSDE by an approximation scheme, along with the decoupling strategy.
\end{abstract}

\section{Keywords}

Non-Markovian, Forward-Backward SDEs, Discontinuous Coefficients, Krylov Estimates, Weak Solution

\section{Introduction}

The following forward-backward stochastic differential equations (FBSDE) on $[t, T]$ are considered in this paper:

$$
\left\{\begin{array}{l}
X_{s}^{t, x}=x+\int_{t}^{s} b\left(r, X_{r}^{t, x}, Y_{r}^{t, x}\right) \mathrm{d} r+\int_{t}^{s} \sigma\left(r, X_{r}^{t, x}, Y_{r}^{t, x}\right) \mathrm{d} W_{r} ; \\
Y_{s}^{t, x}=g\left(X_{T}^{t, x}\right)-\int_{s}^{T} f\left(r, X_{r}^{t, x}, Y_{r}^{t, x}, Z_{r}^{t, x}\right) \mathrm{d} r-\int_{s}^{T} Z_{r}^{t, x} \mathrm{~d} W_{r},
\end{array}\right.
$$

where $W$ is a standard Wiener process and coefficients $b, \sigma, h$ and $g$ are in general random. The coefficient $b$ is allowed to have discontinuity in $y$. More 
precisely, we assume

$$
b(s, x, y)=\sum_{i=1}^{N} b^{i}(s, x) \mathbf{1}_{\left[y_{i-1}, y_{i}\right)}(y), \text { for }(s, x) \in[0, T] \times \mathbb{R}^{n},
$$

and $-\infty<y_{1}<\cdots<y_{N}<\infty$. For simplicity, we shall assume that all the processes involved are 1-dimensional, but as we shall see later, in most of the situation the higher dimensional cases, especially for the forward component $X$ and the Brownian motion $W$, can be argued in an identical way without substantial difficulties.

The FBSDE above is non-Markovian; namely, the coefficients are allowed to be random. The Markovian case (i.e., $b, \sigma, h$ and $g$ are deterministic functions) has been discussed in [3]. For instance, the following is a Markovian-type "regime-switching" FBSDE:

$$
\left\{\begin{array}{l}
X_{t}=x+\int_{0}^{t} b\left(s, X_{s}, Y_{s}\right) \mathrm{d} s+\int_{0}^{t} \sigma\left(s, X_{s}, Y_{s}\right) \mathrm{d} W_{s} \\
Y_{t}=g\left(X_{T}\right)-\int_{t}^{T} h\left(s, X_{s}, Y_{s}, Z_{s}\right) \mathrm{d} s-\int_{t}^{T} Z_{s} \mathrm{~d} W_{s}, \\
X_{0}=x, \quad Y_{T}=g\left(X_{T}\right),
\end{array}\right.
$$

where the coefficients $\sigma, h$, and $g$ are deterministic Lipschitz functions, but the drift coefficient $b$ takes the following form:

$$
b(t, x, y)=\sum_{i=1}^{m} b^{i}(t, x) \mathbf{1}_{\left[a_{i}, a_{i+1}\right)}(y), \quad(t, x, y) \in[0, T] \times \mathbb{R}^{d} \times \mathbb{R},
$$

where $-\infty<a_{1}<a_{2}<\cdots<\infty$ is a finite partition of $\mathbb{R}$, and $b^{i}$ 's are deterministic Lipschitz functions. The main feature of this FBSDE is that the coefficient $b$ has, albeit finitely many, jumps in the variable $Y$. This type of FBSDEs is motivated by the following "regime-switching" term structure model that is often seen in practice. Consider, for example, the Black-Karasinski short rate model that is currently popular in the industry: let $r=\left\{r_{t}: t \geq 0\right\}$ be the short rate process, and $X_{t}=\ln r_{t}, t \geq 0$. Then $X$ satisfies the following SDE:

$$
\mathrm{d} X_{t}=k\left(\theta_{t}-X_{t}\right) \mathrm{d} t+\sigma \mathrm{d} W_{t},
$$

where $W$ is a standard Brownian motion. A simple "regime-switching" version of (5) is that the mean reversion level $\theta$ shifts between two values $\theta_{t} \in\left\{b_{1}, b_{2}\right\}$. The switching in the short-rate is triggered by the level of the long rate. The existence of such structural shift was supported by empirical evidence (see, e.g. [4] [5]). Many dynamic models of the short rate have been proposed, and some of them are hidden Markovian in nature; that is, the switch is triggered by an exogenous factor (diffusion) process $Y$ so that $\theta_{t}=b\left(Y_{t}\right)$, where $b(y) \in\left\{b_{1}, b_{2}\right\}$ (see, e.g., [6] [7] [8] [9]). As a result, "regime switch" models started to attract people's attention. Sometimes business cycles are used as regime classification, while others might define regime by interest rate level. Either way, the important feature of regime switch model remains to be its accommodation to the interactions between regimes and dynamics of the interest rate. A typical approach to model regime switch incorporates a hidden Markov process as a state variable 
into the short rate dynamics. For example,

$$
r_{t+1}-r_{t}=k_{s_{t+1}}\left(\theta_{s_{t+1}}-r_{t}\right)+\sigma_{s_{t+1}} \sqrt{r_{t}} u_{t+1}
$$

(Bansal-Zhou [6])

where $s_{t}$ is a Markov process with given transitional probability, $u_{t+1} \sim N(0,1)$ is the noise. Introduction of the regime-dependence in these papers enriches the flexibility of the model and therefore leads to a higher capacity to fit empirical data.

In particular, if we consider the case in which the triggering process is the long term rate, then following the argument of a term structure model (see, for example, Duffie-Ma-Yong [10]), and assuming the triggering level to be $\alpha>0$ we can derive a FBSDE with discontinuous coefficient:

$$
\left\{\begin{array}{l}
\mathrm{d} X_{t}=\left[b\left(Y_{t}\right)-\beta X_{t}\right] \mathrm{d} t+\sigma \mathrm{d} W_{t} \\
\mathrm{~d} Y_{t}=\left[\mathrm{e}^{X_{t}} Y_{t}-1\right] \mathrm{d} t+Z_{t} \mathrm{~d} W_{t} \\
X_{0}=x, \quad Y_{T}=g\left(X_{T}\right) .
\end{array}\right.
$$

where $X_{t}=\ln r_{t}, Y_{t}$ is the long term treasury bond price; $b(y)=b_{1} \mathbf{1}_{\{y \leq \alpha\}}+b_{2} \mathbf{1}_{\{y>\alpha\}}, \quad b_{1} \neq b_{2}$; and $\alpha, \beta, \sigma$ are constants.

Clearly this is a special case of the FBSDE (3), and its strong solution under Markovian framework has been established in [3]. In this paper, we would like to extend the work of [3] and show the wellposedness of such FBSDE, namely, the existence and uniqueness of a solution $\left(X_{s}^{t, x}, Y_{s}^{t, x}, Z_{s}^{t, x}\right)$ to (2). The paper is organized as follows. In Section 2, we provide necessary preparations, establish assumptions and introduce notations. In section 3 we prove a priori estimates and a stability result. In Section 4 we prove a weak existence of the solution to the FBSDE (2). The main result of this paper is given in Section 5.

\section{Preliminary}

Assumption 2.1. We assume the following standing assumption for this paper.

(A.1) Each $b^{i}(s, x)$ is bounded, continuous in $s$, and uniformly Lipschitz in $x$, with Lipschitz constant $K>0$;

(A.2) The function $\sigma(s, x, y)$ is continuous, and there exist constants $0<\underline{\sigma}<\bar{\sigma}$ such that $\underline{\sigma} \leq \sigma(s, x, y) \leq \bar{\sigma}$. Furthermore, for fixed $s, \sigma(s, \cdot, \cdot)$, along with its spatial derivatives, $\sigma_{x}$ and $\sigma_{y}$ are all uniformly Lipschitz in $(x, y)$, with Lipschitz constant $K>0$;

(A.3) The function $f(s, x, y, z)$ is bounded, continuous in $s$ and uniformly Lipschitz in $(x, y, z)$, with Lipschitz constant $K>0$;

(A.4) The function $g(x)$ is bounded, smooth and Lipschitz in $X$, and $I_{0} \triangleq \mathbb{E}\left\{\|g(\cdot)\|_{0}^{2}+\int_{0}^{T}\|f(s, ;, 0,0)\|_{0}^{2} \mathrm{~d} s\right\}<\infty$, where the norm $\|\cdot\|_{0}$ is define in (11).

It is well-understood that, in order to solve a fully coupled FBSDE one should look for a "decoupling random field" $u(t, x, \omega)$, such that $Y_{t} \equiv u\left(t, X_{t}\right)$, for all $t \in[0, T], \mathbb{P}$-a.s. (cf. e.g., [11]). In the Non-Markovian case, the decoupling 
field should be a random field $u: \times[0, T] \times \mathbb{R} \mapsto \mathbb{R}$, and in light of the stochastic Feynman-Kac formula (cf. [12]), we expect that such a function $u$ should solve the following quasilinear BSPDEs, in a certain sense:

$$
\left\{\begin{array}{l}
\mathrm{d} u=\alpha(s, x) \mathrm{d} s+\beta(s, x) \mathrm{d} W_{s} \\
u(T, x)=g(x)
\end{array}\right.
$$

where

$$
\left\{\begin{array}{l}
\alpha(s, x)=-\left\{\frac{1}{2} u_{x x} \sigma^{2}(s, x, u)+\beta_{x} \sigma(s, x, u)+u_{x} b(s, x, u)+f(s, x, u, \gamma)\right\} \mathrm{d} s \\
\gamma(s, x)=\beta(s, x)+u_{x}(s, x) \sigma(s, x, u(s, x)) .
\end{array}\right.
$$

We should note that a solution to the BSPDE is defined as the pair of progressively measurable random fields $(u, \beta)$. Clearly, when the coefficients are deterministic, we must have $\beta=0$ and the $\operatorname{BSPDE}(7)$ is reduced to a quasilinear PDE.

We next introduce the notion of the weighted Sobolev space. We begin by considering a function $\phi \in \mathbb{C}^{\infty}(\mathbb{R})$ that satisfies the following conditions:

$$
\left\{\begin{array}{l}
0<\phi(x) \leq 1, \quad \int_{\mathbb{R}} \phi(x) \mathrm{d} x=1 ; \\
\phi(x)=\mathrm{e}^{-|x|} \text { for } x \text { large enough. }
\end{array}\right.
$$

We shall call such a smooth function $\phi$ the weight function. One can easily check that if $\phi$ is a weight function, then one has

$$
K_{\phi} \triangleq \sup _{x \in \mathbb{R}}|D \phi(x)|+\left|D^{2} \phi(x)\right| \phi(x)<\infty .
$$

Now for a given weight function $\phi$, we denote $H_{\phi}^{0}(\mathbb{R} ; \mathbb{R})$ to be the space of all Lebesgue measurable functions $h: \mathbb{R} \rightarrow \mathbb{R}$ such that $\|h\|_{0}^{2} \int_{\phi}|h(x)|^{2} \phi(x) \mathrm{d} x<\infty$. When the weight function and the dimension of the domain and range spaces are clear from the context, and there is no danger of confusion, we often drop the subscript $\phi$ and the spaces in the notation, and denote simply as $H^{0}$. Clearly $H^{0}$ is a Hilbert space equipped with the following inner product:

$$
\left\langle h^{1}, h^{2}\right\rangle_{0} \triangleq \int_{\mathbb{R}} h^{1}(x) h^{2}(x) \phi(x) \mathrm{d} x .
$$

We can now define the Weighted Sobolev spaces as usual. For example, we shall denote $H^{1}=H_{\phi}^{1} \subset H^{0}$ to be the subspace of $H^{0}$ that consists of all those $h$ such that its generalized derivative, still denoted as $h_{x}$, is also in $H^{0}$. Clearly, $H^{1}$ is a Hilbert space with the inner product $\left\langle h^{1}, h^{2}\right\rangle_{1} \triangleq\left\langle h^{1}, h^{2}\right\rangle_{0}+\left\langle h_{x}^{1}, h_{x}^{2}\right\rangle_{0}$, where $h^{1}, h^{2} \in H^{1}$. One can easily prove the integration by parts formula: for any $h^{1} \in H^{1}(\mathbb{R}, \mathbb{R})$ and $h^{2} \in H^{1}(\mathbb{R}, \mathbb{R})$,

$$
\left\langle h_{x}^{1}, h^{2}\right\rangle_{0}=-\left\langle h^{1}, h_{x}^{2}\right\rangle_{0}-\left\langle h^{1} h^{2}, \frac{\phi_{x}}{\phi}\right\rangle_{0} .
$$

Similarly, we denote $H^{2}=H_{\phi}^{2} \subset H^{1}$ to be the subspace of $H^{1}$ that contains all $h \in H^{0}$ such that $h_{x} \in H^{1}$. Thus, $H^{2}$ is again a Hilbert space with inner product $\left\langle h^{1}, h^{2}\right\rangle_{2} \triangleq\left\langle h^{1}, h^{2}\right\rangle_{0}+\left\langle h_{x}^{1}, h_{x}^{2}\right\rangle_{1}$, where $h^{1}, h^{2} \in H^{2}$. Moreover, let $H^{-1}$ 
be the dual space of $H^{1}$, endowed with the dual product $\langle\cdot,\rangle_{-1}$. Then $H^{-1}$ is equipped with the following norm: $\|h\|_{-1} \triangleq \sup \left\{\langle h, \varphi\rangle_{-1}: \varphi \in H^{1},\|\varphi\|_{1}=1\right\}$. Clearly, $H^{0} \subset H^{-1}$ in the sense that for any $\alpha \in H^{0}$, it holds that

$$
\langle\alpha, \varphi\rangle_{-1}=\langle\alpha, \varphi\rangle_{0}, \quad \forall \varphi \in H^{1} .
$$

Furthermore, for any $h \in H^{0}(\mathbb{R}, \mathbb{R})$, in light of (12), we have $h_{x} \in H^{-1}$ in the following sense: for any $\varphi \in H^{1}(\mathbb{R}, \mathbb{R}),\left\langle h_{x}, \varphi\right\rangle_{-1}-\left\langle h, \varphi_{x}\right\rangle_{0}-\left\langle h \varphi, \frac{\phi_{x}}{\phi}\right\rangle_{0}$.

Remark 2.2. It is worth noting that

1) For any two weight functions $\phi_{1}, \phi_{2}$ satisfying (9), there must exist constants $0<c<C$ such that $c \phi_{1} \leq \phi_{2} \leq C \phi_{1}$. So the norms defined via $\phi_{1}$ and $\phi_{2}$ are equivalent, and therefore, the spaces $H^{i}, i=-1,0,1,2$, are independent of the choices of $\phi$.

2) It is readily seen that the weight function belongs to the class of the so-called Schwartz functions, and consequently any functions with polynomial growth are in $H^{0}$.

We conclude this section by introducing some spaces of stochastic processes that will be useful for the study of the BSPDEs. First, for any sub- $\sigma$-field $\mathcal{G} \subseteq \mathcal{F}$, and $0 \leq p \leq \infty$, we denote $L^{p}(\mathcal{G})$ to be the spaces of all $\mathcal{G}$-measurable, $L^{p}$ -integrable random variables. Next, for any generic Banach space $\mathbb{B}$, we denote $L_{\mathcal{F}}^{p}([0, T] ; \mathbb{B})$ to be all $\mathbb{B}$-valued, $\mathbb{F}$-progressively measurable random fields (or processes) $h:[0, T] \mapsto \mathbb{B}$ such that

$$
\|h\|_{L_{\mathcal{F}}^{p}([0, T] ; \mathbb{B})} \triangleq \mathbb{E}\left\{\int_{0}^{T}\|h(s, \cdot)\|_{\mathbb{B}}^{p} \mathrm{~d} s\right\}^{1 / p}<\infty .
$$

In particular, if $\mathbb{B}=H_{\phi}^{i}$, where $\phi$ is a given weight function, we denote $\mathscr{S}_{\phi}^{i}=L_{\mathbb{F}}^{2}\left([0, T] ; H_{\phi}^{i}\right), \quad i=-1,0,1,2$, respectively. Again, we often drop the subscript $\phi$ from the notations when the context is clear. Finally, the spaces of Banach-space-valued processes such as $\mathbb{C}_{\mathbb{F}}^{\alpha}([0, T] ; \mathbb{B})$, for $\alpha \geq 1$, are defined in the obvious way.

We now define the notion of Sobolev weak solutions to BSPDE (7).

Definition 2.1. We say that the pair of random fields $(u, \beta) \in \mathscr{C}^{1} \times \mathscr{C}^{0}$ is a weak solution to BSPDE (7) if $D \sigma(s, x, u(s, x))$ is uniformly bounded and, for any $\varphi \in H^{1}$, it holds that

$$
\mathrm{d}\langle u(s, \cdot), \varphi\rangle_{0}=\langle\alpha(s, \cdot), \varphi\rangle_{-1} \mathrm{~d} s+\langle\beta(s, \cdot) \mathrm{d} W(s), \varphi\rangle_{0}, \text { a.s. }
$$

We say that $(u, \beta) \in \mathscr{K}^{1} \times \mathscr{K}^{0}$ is a regular weak solution to BSPDE (7) if $(u, \beta)$ is a weak solution such that $D u$ is uniformly bounded.

\section{A Priori Estimates and a Stability Result}

Proposition 3.1. Assume Assumption 2.1. Let $(u, \beta) \in \mathscr{H}^{1} \times \mathscr{H}^{0}$ be a weak solution to the BSPDE (7). Then there exists a constant $C>0$, depending only on the bounds in Assumption 2.1, the duration $T$ and the constant $K_{\phi}$ for the given weight function (10), such that 


$$
\mathbb{E}\left\{\sup _{s \in[0, T]}\|u(s, \cdot)\|_{0}^{2}+\int_{0}^{T}\|\gamma(s, \cdot)\|_{0}^{2} \mathrm{~d} s\right\} \leq C\left[1+I_{0}\right]
$$

Proof. For simplicity, let us denote $\tilde{\phi} \triangleq \frac{\phi_{x}}{\phi}$. Also let us denote $f(s, x, u, \gamma)$ as $f$. By integration by parts formula and a general Itô formula (see [13]), one has,

$\mathrm{d}\|u(s, x)\|_{0}^{2}=\left\{-2\langle\alpha, u\rangle_{-1}+\|\beta\|_{0}^{2}\right\} \mathrm{d} s+2\langle u, \beta \mathrm{d} W\rangle_{0}$

$=\int_{\mathbb{R}}\left[u_{x}\left(u \sigma^{2} \phi\right)_{x}+2 \beta(u \sigma \phi)_{x}-2 u u_{x} b \phi-2 u f \phi+\beta^{2} \phi\right] \mathrm{d} x \mathrm{~d} s+2\langle u, \beta \mathrm{d} W\rangle_{0}$

$=\int_{\mathbb{R}}\left[\gamma^{2} \phi+2 u\left[\sigma_{x} \phi+\sigma \phi_{x}\right] \gamma-u u_{x} \sigma^{2} \phi_{x}-2 u u_{x} b \phi-2 u f \phi\right] \mathrm{d} x \mathrm{~d} s+2\langle u, \beta \mathrm{d} W\rangle_{0}$

$=\int_{\mathbb{R}}\left[\gamma^{2}+2 u\left[\sigma_{x}+\sigma \tilde{\phi}\right] \gamma+u^{2}\left[12 \sigma^{2} \tilde{\phi} \phi\right]_{x} / \phi-2 u u_{x} b-2 u f\right] \phi \mathrm{d} x \mathrm{~d} s+2\langle u, \beta \mathrm{d} W\rangle_{0}$.

There is only one troubled term in the above equality needs special treatment, $2 u u_{x} b \phi$. For this purpose, we define $B(x, z) \triangleq \int_{0}^{z} y b(x, y) \mathrm{d} y$. Taking the derivative with respect to $x$ to get $\frac{\partial}{\partial x} B(x, u)=\int_{0}^{u} y b_{x}(x, y) \mathrm{d} y+u u_{x} b(x, u)$. Hence

$$
\begin{aligned}
-\int_{\mathbb{R}} 2 u u_{x} b \phi \mathrm{d} x & =-\int_{\mathbb{R}} 2 \frac{\partial}{\partial x} B(x, u) \phi \mathrm{d} x+\int_{\mathbb{R}} \int_{0}^{u} 2 y b_{x}(x, y) \phi \mathrm{d} y \mathrm{~d} x \\
& \geq \int_{\mathbb{R}} 2 B(x, u) \phi_{x} \mathrm{~d} x-C \int_{\mathbb{R}} \int_{0}^{|u|} y \mathrm{~d} y \mathrm{~d} x \\
& \geq \int_{\mathbb{R}} \int_{0}^{u} 2 y b(x, y) \phi_{x} \mathrm{~d} y \mathrm{~d} x-C \int_{\mathbb{R}} u^{2} \mathrm{~d} x \\
& \geq-C \int_{\mathbb{R}} u^{2} \mathrm{~d} x,
\end{aligned}
$$

where $C$ is the generic constant described in the statement of the Proposition. Thus (17) becomes

$$
\begin{aligned}
& \mathrm{d}\|u(s, x)\|_{0}^{2} \geq \int_{\mathbb{R}}\left[12 \gamma^{2}-C u^{2}-\frac{1}{16(K+1)^{2}} f^{2}\right] \phi \mathrm{d} x \mathrm{~d} s+2\langle u, \beta \mathrm{d} W\rangle_{0} \\
& \geq \int_{\mathbb{R}}\left[12 \gamma^{2}-C u^{2}-\frac{1}{8(K+1)^{2}}\left\{f^{2}(s, x, 0,0)+|f-f(s, x, 0,0)|^{2}\right\}\right] \phi \mathrm{d} x \mathrm{~d} s \\
& +2\langle u, \beta \mathrm{d} W\rangle_{0} \\
& \geq \int_{\mathbb{R}}\left[12 \gamma^{2}-C u^{2}-\frac{1}{8(K+1)^{2}}\left\{f^{2}(s, x, 0,0)+2 K^{2} u^{2}\right\}-\frac{1}{4} \gamma^{2}\right] \phi \mathrm{d} x \mathrm{~d} s \\
& +2\langle u, \beta \mathrm{d} W\rangle_{0}
\end{aligned}
$$

The proof can be completed by integrating the above inequality from $s$ to $T$, and applying the Gronwall inequality.

Proposition 3.2. Let $(b, \sigma, f, g)$ and $\left(b^{(l)}, \sigma^{(l)}, f^{(l)}, g^{(l)}\right), l=1,2, \cdots \quad$ be a sequence of coefficients of BSPDE (7) satisfying Assumption 2.1 uniformly. Assume that
1) $\lim _{l \rightarrow \infty}\left[\left\|b^{(l)}-b\right\|_{\mathscr{\ell}^{0}}^{2}+\left\|\sigma^{(l)}-\sigma\right\|_{\mathscr{C}^{1}}^{2}+\left\|g^{(l)}-g\right\|_{\not \ell^{0}}^{2}\right]=0$;
2) For any fixed $(x, y, z), \lim _{l \rightarrow \infty} \mathbb{E}\left\{\int_{0}^{T}\left|f^{(l)}-f\right|^{2}(s, x, y, z) \mathrm{d} s\right\}=0$; 
3) For each 1 , BSPDE (7) with coefficients $\left(b^{(l)}, \sigma^{(l)}, f^{(l)}, g^{(l)}\right)$ has a weak solution $\left(u^{(l)}, \beta^{(l)}\right)$. The solutions $u^{(l)}$ are uniformly bounded in supremum norm and $u_{x}^{(l)}$ are uniformly bounded in $\mathscr{C}^{0}$, uniformly in $l$;

4) There exists $u \in \mathscr{K}^{0}$ such that $\lim _{l \rightarrow \infty}\left\|u^{(l)}-u\right\|_{\mathscr{\not} 0}^{2}=0$.

Then $u \in \mathscr{C}^{1}$ and there exists $\beta \in \mathscr{C}^{0}$ such that $(u, \beta)$ is a weak solution to BSPDE (7) with coefficients $(b, \sigma, f, g)$.

Proof. By the definition of $\mathscr{K}^{0}, \phi$ and condition 3$), u_{x}^{(l)}$ are uniformly bounded in $\mathscr{H}^{0}$. So there exists $v \in \mathscr{H}^{0}$ such that $u_{x}^{(l)} \rightarrow v$ weakly in $\mathscr{H}^{0}$. It is clear that $v$ is bounded. Moreover, the differential operator with respect to $x$ is a closed operator, that is, for any $h \in \mathscr{H}^{1}$,

$$
\langle v, h\rangle_{0}=\lim _{l \rightarrow \infty}\left\langle u_{x}^{(l)}, h\right\rangle_{0}=\lim _{l \rightarrow \infty}\left\langle u^{(l)}, h_{x}\right\rangle_{0}=\left\langle u, h_{x}\right\rangle_{0} .
$$

This implies that $u_{x}=v$. Hence $u \in \mathscr{H}^{1}$ and $u_{x}$ is bounded in $\mathscr{C}^{0}$. Next, denote $\gamma^{(l)} \triangleq \beta^{(l)}+u_{x}^{(l)} \sigma^{(l)}$. By Proposition 3.1, we know $\left\|\gamma^{(l)}\right\|_{\not{ }^{0}} \leq C$ uniformly in $I$ for some constant $C$. Thus we can extract a subsequence, still indexed by $(I)$, such that

$$
\lim _{l \rightarrow \infty}\left\langle\gamma^{(l)}-\gamma, h\right\rangle_{\gamma^{0}}=0
$$

for some $\gamma \in \mathscr{H}^{0}$ and any $h \in \mathscr{H}^{0}$. Note that by the boundedness of $u_{x}^{(l)}$ and $\sigma^{(l)}$, we know that $\left\|\beta^{(l)}\right\|_{\mathscr{\gamma}^{0}} \leq C$ uniformly in 1 . We now define $\beta \triangleq \gamma-u_{x} \sigma$. Note that, for any $h \in \mathscr{C}^{0}$,

$$
\begin{aligned}
& \lim _{l \rightarrow \infty}\left\langle\beta^{(l)}, h\right\rangle_{\mathscr{R}^{0}} \\
& =\lim _{l \rightarrow \infty}\left\langle\gamma^{(l)}-u_{x}^{(l)} \sigma^{(l)}, h\right\rangle_{\not^{0}} \\
& =\lim _{l \rightarrow \infty}\left\{\left\langle\gamma^{(l)}, h\right\rangle_{\not \gamma^{0}}-\left\langle u_{x}^{(l)} \sigma, h\right\rangle_{\not \gamma^{0}}+\left\langle u_{x}^{(l)}\left[\sigma-\sigma^{(l)}\right], h\right\rangle_{\not y^{0}}\right\} \\
& =\langle\gamma, h\rangle_{\not \gamma^{0}}-\left\langle u_{x} \sigma, h\right\rangle_{\not \gamma 0}+0 \\
& =\langle\beta, h\rangle_{\not 0},
\end{aligned}
$$

where the second convergence is due to (19) and the boundedness of $\sigma$, and the third convergence is due to the uniform boundedness of $u_{x}^{(l)}$. That is, $\beta^{(l)}$ converges to $\beta$ weakly in $\mathscr{K}^{0}$. It remains to show that $(u, \beta)$ is a weak solution to BSPDE (7) with coefficients $(b, \sigma, f, g)$.

It suffices to check (15). We fix $t \leq t_{1}<t_{2} \leq T$ and a smooth function $\varphi$ with compact support. For each $l$, since $\left(u^{(l)}, \beta^{(l)}\right)$ is a weak solution to the corresponding BSPDE, we have

$$
\begin{aligned}
& \left\langle u^{(l)}\left(t_{2}, \cdot\right)-u^{(l)}\left(t_{1}, \cdot\right), \varphi\right\rangle_{0} \\
& =\int_{t_{1}}^{t_{2}}\left\langle\alpha^{(l)}(s, \cdot), \varphi\right\rangle_{-1} \mathrm{~d} s+\int_{t_{1}}^{t_{2}}\left\langle\beta^{(l)}(s, \cdot) \mathrm{d} W(s), \varphi\right\rangle_{0} \\
& =\int_{t_{1}}^{t_{2}} \int_{\mathbb{R}} \alpha^{(l)}(s, x) \varphi(x) \phi(x) \mathrm{d} x \mathrm{~d} s+\int_{t_{1}}^{t_{2}}\left\langle\beta^{(l)}(s, \cdot) \mathrm{d} W(s), \varphi\right\rangle_{0}
\end{aligned}
$$




$$
\begin{aligned}
= & \int_{t_{1}}^{t_{2}} \int_{\mathbb{R}}\left\{\frac{1}{2} u_{x}^{(l)}\left[\left(\sigma^{(l)}\right)^{2} \varphi \phi\right]_{x}+\beta^{(l)}\left[\sigma^{(l)} \varphi \phi\right]_{x}-u_{x}^{(l)} b^{(l)} \varphi \phi-f^{(l)} \varphi \phi\right\} \mathrm{d} x \mathrm{~d} s \\
& +\int_{t_{1}}^{t_{2}}\left\langle\beta^{(l)}(s, \cdot) \mathrm{d} W(s), \varphi\right\rangle_{0} \\
= & \int_{t_{1}}^{t_{2}} \int_{\mathbb{R}}\left\{\left[u_{x}^{(l)} \sigma^{(l)}+\beta^{(l)}\right]\left[\sigma_{x}^{(l)}+u_{x}^{(l)} \sigma_{y}^{(l)}\right] \varphi \phi\right. \\
& +\left[\frac{1}{2} u_{x}^{(l)}\left(\sigma^{(l)}\right)^{2}+\beta^{(l)} \sigma^{(l)}\right]\left[\varphi_{x} \phi+\varphi \phi_{x}\right] \\
& \left.-\left[u_{x}^{(l)} b^{(l)}+f^{(l)}\right] \varphi \phi\right\} \mathrm{d} x \mathrm{~d} s+\int_{t_{1}}^{t_{2}}\left\langle\beta^{(l)}(s, \cdot) \mathrm{d} W(s), \varphi\right\rangle_{0} .
\end{aligned}
$$

Note that since the boundedness and the convergence properties of all the involved terms, and thanks to Assumption 2.1, there is only one term left to check, $\int_{t_{1}}^{t_{2}} \int_{\mathbb{R}} u_{x}^{(l)} b^{(l)} \varphi \phi \mathrm{d} x \mathrm{~d} s$. To this end, we define $B(x, z) \triangleq \int_{0}^{z} b(x, y) \mathrm{d} y$ and $B^{(l)}(x, z) \triangleq \int_{0}^{z} b^{(l)}(x, y) \mathrm{d} y$. Clearly $B^{(l)}(x, z)$ and $B(x, z)$ are continuous and $B^{(l)}(x, z)$ converges to $B(x, z)$ pointwise. Since $b, b^{(l)}, u^{(l)}$ are bounded, say, by a constant $M>0$, we have

$$
\begin{aligned}
& \left|B^{(l)}\left(x, u^{(l)}\right)-B(x, u)\right| \\
& =\left|\int_{0}^{u^{(l)}} b^{(l)}(x, y) \mathrm{d} y-\int_{0}^{u} b(x, y) \mathrm{d} y\right| \\
& \leq\left|\int_{0}^{u^{(l)}}\left(b^{(l)}(x, y)-b(x, y)\right) \mathrm{d} y\right|+\left|\int_{u^{(l)}}^{u} b(x, y) \mathrm{d} y\right| \\
& \leq \int_{-M}^{M}\left|b^{(l)}(x, y)-b(x, y)\right| \mathrm{d} y+\left|\int_{u^{(l)}}^{u} M \mathrm{~d} y\right| .
\end{aligned}
$$

From this it is easy to see that $B^{(l)}\left(x, u^{(l)}\right)$ converges to $B(x, u)$, and the limit is uniform in $(s, x)$ on any compact subset of $[0, T) \times \mathbb{R}$. Using similar argument one can show that

$$
\int_{\mathbb{R}} \int_{0}^{u^{(l)}} b_{x}^{(l)}(x, y) \varphi \phi \mathrm{d} y \mathrm{~d} x \rightarrow \int_{\mathbb{R}} \int_{0}^{u} b_{x}(x, y) \varphi \phi \mathrm{d} y \mathrm{~d} x .
$$

Similar to Proposition 3.1, one gets

$$
\begin{aligned}
& \lim _{l \rightarrow \infty} \int_{\mathbb{R}} u_{x}^{(l)} b^{(l)}\left(x, u^{(l)}\right) \varphi \phi \mathrm{d} x \\
& =\lim _{l \rightarrow \infty} \int_{\mathbb{R}}\left[\frac{\partial}{\partial x} B^{(l)}\left(x, u^{(l)}\right)-\int_{0}^{u^{(l)}} b_{x}^{(l)}(x, y) \mathrm{d} y\right] \varphi \phi d x \\
& =-\lim _{l \rightarrow \infty} \int_{\mathbb{R}} B^{(l)}\left(x, u^{(l)}\right)[\varphi \phi]_{x} \mathrm{~d} x-\lim _{l \rightarrow \infty} \int_{\mathbb{R}} \int_{0}^{u^{(l)}} b_{x}^{(l)}(x, y) \varphi \phi \mathrm{d} y \mathrm{~d} x \\
& =-\int_{\mathbb{R}} B(x, u)[\varphi \phi]_{x} \mathrm{~d} x-\int_{\mathbb{R}} \int_{0}^{u} b_{x}(x, y) \varphi \phi \mathrm{d} y \mathrm{~d} x \\
& =\int_{\mathbb{R}}\left[\frac{\partial}{\partial x} B(x, u)-\int_{0}^{u} b_{x}(x, y) \mathrm{d} y\right] \varphi \phi \mathrm{d} x=\int_{\mathbb{R}} u_{x} b(x, u) \varphi \phi \mathrm{d} x,
\end{aligned}
$$

and this completes the proof.

\section{Weak Existence}

Parallel to the Itô-Krylov formula ([14], Theorem 2.10.1), we provide a general version of Itô-Ventzell formula which requires weaker regularity conditions. The 
proof can be easily produced similarly to the proof of Itô-Krylov formula. The key point in the proof is the utilization of the boundedness and non-degeneracy assumptions of the coefficients. We refer the readers Theorem 2.10.1 of [14], and omit the proof.

Lemma 4.1. Consider the process

$$
\xi(s)=x+\int_{0}^{s} a(r) \mathrm{d} r+\int_{0}^{s} h(r) \mathrm{d} W_{r} .
$$

Let $Q$ be a bounded region in $[0, T] \times \mathbb{R}$ and $\tau_{Q}$ be the first exit time of the process $\xi$ from the region $Q$. Let $\tau$ be some Markov time such that $\tau \leq \tau_{Q}$. Assume further that there exist positive constants $C_{1}$ and $C_{2}$, such that $\left|a_{s}\right|+\left|h_{s}\right| \leq C_{1}$ and $\left|h_{s}\right| \geq C_{2}$ for all $\omega$ and $t<\tau$. Then for any Itô process $F$ such that $F \in W^{1,2}(Q)$ for $\mathbb{P}$-a.s. $\omega$, and

$$
\mathrm{d} F(s, \omega, x)=A(s, \omega, x) \mathrm{d} s+H(s, \omega, x) \mathrm{d} W_{s},
$$

where $A \in \mathscr{K}^{0}$ and $H \in \mathscr{H}^{1}$, the following holds:

$$
\begin{aligned}
& F(\tau, \xi)-F(s, \xi) \\
& =\int_{s}^{\tau}\left\{A(r, \xi)+a(r) F_{x}(r, \xi)+h(r) H_{x}(r, \xi)+\frac{1}{2} h^{2}(r) F_{x x}(r, \xi)\right\} \mathrm{d} r \\
& \quad+\int_{s}^{\tau}\left\{H(r, \xi)+h(r) F_{x}(r, \xi)\right\} \mathrm{d} W_{r} .
\end{aligned}
$$

Now we are ready to show the weak existence of FBSDE (2). Let us first introduce some notations. For any $N \in \mathbb{N}$, let $t=t_{0}<t_{1}<\cdots<t_{N}=T$ be the partition on $[t, T]$ where $t_{i}=t+\frac{i(T-t)}{2^{N}}, i=0, \cdots, 2^{N}$. For any $\mathbf{x}=\left(x_{1}, x_{2}, \cdots, x_{N}\right) \in \mathbb{R}^{N}$, and $k=1,2, \cdots, N$, we denote

$$
\mathbf{x}^{(k)} \triangleq\left(x_{1}, \cdots, x_{k}\right) \text { and } W_{s}^{(k)} \triangleq\left(W_{t_{1} \wedge s}, \cdots, W_{t_{k} \wedge s}\right) .
$$

Let $b_{N}(s, \omega, x, y) \triangleq E\left[b(s, \cdot, x, y) \mid \mathcal{F}_{s}^{N}\right]$, where $\mathcal{F}_{s}^{N}$ is the filtration generated by $W_{s}^{(N)}$, and clearly for fixed $(s, x, y)$,

$$
b_{N}(s, x, y)-b(s, x, y) \rightarrow 0 \quad \mathbb{P} \text {-a.s., as } N \rightarrow \infty .
$$

Similarly we define $\sigma_{N}, f_{N}$, and $g_{N}$. Clearly $b_{N}$ can be written in terms of $\left(s, W_{s}^{(N)}, x, y\right)$ in the canonical space. Let us denote

$$
\tilde{b}_{N}\left(s, W_{s}^{(N)}, x, y\right) \triangleq b_{N}(s, \omega, x, y) .
$$

Then $\tilde{b}_{N}\left(s, \mathbf{x}^{(N)}, x, y\right)$ is a deterministic function from $[0, T] \times \mathbb{R}^{N+2}$ to $\mathbb{R}$. Similarly, we define $\tilde{\sigma}_{N}, \tilde{f}_{N}$, and $\tilde{g}_{N}$.

Proposition 4.2. Under Assumption 2.1, the FBSDE (2) admits a weak solution.

Proof. Step 1 (Weak Existence of the BSPDE): In Step 1 and Step 2, we assume that for all $N, \tilde{b}_{N}, \tilde{\sigma}_{N}, \tilde{f}_{N}$ and $\tilde{g}_{N}$ are smooth functions except for the $y$ component of $\tilde{b}_{N}$. We consider the following FBSDE:

$$
\left\{\begin{array}{l}
\bar{X}_{N, s}^{t, x}=\bar{X}_{0}+\int_{t}^{s} \bar{b}_{N}\left(r, \bar{X}_{N, r}^{t, x}, Y_{N, s}^{t, x}\right) \mathrm{d} r+\int_{t}^{s} \bar{\sigma}_{N}\left(r, \bar{X}_{N, r}^{t, x}, Y_{N, s}^{t, x}\right) \mathrm{d} W(r) ; \\
Y_{N, s}^{t, x}=\tilde{g}_{N}\left(\bar{X}_{N, T}^{t, x}\right)+\int_{s}^{T} \tilde{f}_{N}\left(r, \bar{X}_{N, r}^{t, x}, Y_{N, r}^{t, x}, Z_{N, r}^{t, x}\right) \mathrm{d} r-\int_{s}^{T} Z_{N, r}^{t, x} \mathrm{~d} W(r),
\end{array}\right.
$$


where

$$
\bar{X}_{0} \triangleq\left(\begin{array}{c}
0 \\
\vdots \\
0 \\
x
\end{array}\right), \bar{b}_{N}(s, \mathbf{x}, y) \triangleq\left(\begin{array}{c}
0 \\
\vdots \\
0 \\
\tilde{b}_{N}(s, \mathbf{x}, y)
\end{array}\right) \text { and } \bar{\sigma}_{N}(s, \mathbf{x}, y) \triangleq\left(\begin{array}{c}
1_{\left\{s \leq t_{1}\right\}} \\
\vdots \\
1_{\left\{s \leq t_{N}\right\}} \\
\tilde{\sigma}_{N}(s, \mathbf{x}, y)
\end{array}\right)
$$

for any $(s, \mathbf{x}, y) \in[0, T] \times \mathbb{R}^{N+2}$. Because of the assumption of smoothness, FBSDE (22) is a deterministic FBSDE, and by Theorem 5.1 in [3], FBSDE (22) admits a unique strong solution $\left(\bar{X}_{N, s}^{t, x}, Y_{N, s}^{t, x}, Z_{N, s}^{t, x}\right)$. Indeed, $\bar{X}_{N, s}^{t, x}=\left(W_{s}^{(N)}, X_{N, s}^{t, x}\right)$, where $\left(X_{N, s}^{t, x}, Y_{N, s}^{t, x}, Z_{N, s}^{t, x}\right)$ is the unique strong solution (again by Theorem $5.1 \mathrm{in}$ [3]) of

$$
\left\{\begin{array}{l}
X_{N, s}^{t, x}=x+\int_{t}^{s} \tilde{b}_{N}\left(r, W_{r}^{(N)}, X_{N, r}^{t, x}, Y_{N, s}^{t, x}\right) \mathrm{d} r+\int_{t}^{s} \tilde{\sigma}_{N}\left(r, W_{r}^{(N)}, X_{N, r}^{t, x}, Y_{N, s}^{t, x}\right) \mathrm{d} W(r) \\
Y_{N, s}^{t, x}=\tilde{g}_{N}\left(W_{T}^{(N)}, X_{N, T}^{t, x}\right)+\int_{s}^{T} \tilde{f}_{N}\left(r, W_{r}^{(N)}, X_{N, r}^{t, x}, Y_{N, r}^{t, x}, Z_{N, r}^{t, x}\right) \mathrm{d} r-\int_{s}^{T} Z_{N, r}^{t, x} \mathrm{~d} W(r) .
\end{array}\right.
$$

Now let us define a function $\tilde{u}_{N}$ piecewisely, and this function is essential in finding the decoupling field of FBSDE (2). First for any $\mathbf{x}^{(N)} \in \mathbb{R}^{N}$ and $x \in \mathbb{R}$, define

$$
\tilde{u}_{N, N}\left(T, \mathbf{x}^{(N)}, x\right)=\tilde{g}_{N}\left(\mathbf{x}^{(N)}, x\right) .
$$

Secondly, for each $k=N, N-1, \cdots, 1$, we consider the FBSDE (22) on $\left[t_{k-1}, t_{k}\right) \times \mathbb{R}^{k} \times \mathbb{R}$. By Theorem 5.1 in [3], for any $\left(s, \mathbf{x}^{(k)}, x\right) \in\left[t_{k-1}, t_{k}\right) \times \mathbb{R}^{k} \times \mathbb{R}$, there exists a deterministic decoupling field $\tilde{u}_{N}\left(s, \mathbf{x}^{(k)}, x\right)$, which is a weak solution to the corresponding PDE, such that

$$
Y_{N, s}^{t, x}=\tilde{u}_{N, k}\left(s, W_{s}^{(k)}, X_{N, s}^{t, x}\right) .
$$

Furthermore, let us patch functions $\tilde{u}_{N, k}$ 's together by defining the following function $\tilde{u}_{N}$ :

$$
\left\{\begin{array}{l}
\tilde{u}_{N}\left(T, \mathbf{x}^{(N)}, x\right) \triangleq \tilde{u}_{N, N}\left(T, \mathbf{x}^{(N)}, x\right) ; \\
\tilde{u}_{N}\left(s, \mathbf{x}^{(N)}, x\right) \equiv \tilde{u}_{N}(s, \mathbf{x}^{(k-1)}, \underbrace{x_{k}, \cdots, x_{k}}_{N-k+1}, x) \triangleq \tilde{u}_{N, k}\left(s, \mathbf{x}^{(k)}, x\right), \text { if } s \in\left[t_{k-1}, t_{k}\right) .
\end{array}\right.
$$

Thus on $[t, T]$, we have

$$
Y_{N, s}^{t, x}=\tilde{u}_{N}\left(s, W_{s}^{(N)}, X_{N, s}^{t, x}\right) .
$$

For any $s \in[0, T]$, denote $\theta_{s} \triangleq\left(s, W_{s}^{(N)}, x\right)$ and $\Theta_{N, s}^{t} \triangleq\left(s, W_{s}^{(N)}, X_{N, s}^{t, x}\right)$. Note that since all coefficients are bounded, we know that second derivatives of $\tilde{u}_{N} \in W_{2}^{1,2}$ (see [15]). Here $W_{2}^{1,2}=\left\{u: u, u_{t}, u_{x}, u_{x x} \in L^{2}\right\}$. By the Itô-Krylov formula, for $s \in\left[t_{k-1}, t_{k}\right)$ and fixed $x$, one has

$$
\mathrm{d} \tilde{u}_{N}\left(\theta_{s}\right)=\left\{\tilde{u}_{N s}\left(\theta_{s}\right)+\frac{1}{2} \sum_{i=1}^{k} \tilde{u}_{N x_{i} x_{i}}\left(\theta_{s}\right)\right\} \mathrm{d} s+\sum_{i=1}^{k} \tilde{u}_{N x_{i}}\left(\theta_{s}\right) \mathrm{d} W(s) .
$$

Thus we define 


$$
\left\{\begin{array}{l}
\tilde{\alpha}_{N, k}\left(s, \mathbf{x}^{(k)}, x\right) \triangleq \tilde{u}_{N s}\left(s, \mathbf{x}^{(k)}, x\right)+\frac{1}{2} \sum_{i=1}^{k} \tilde{u}_{N x_{i} x_{i}}\left(s, \mathbf{x}^{(k)}, x\right), \text { for } s \in\left[t_{k-1}, t_{k}\right) ; \\
\tilde{\alpha}_{N}\left(s, \mathbf{x}^{(N)}, x\right) \triangleq \tilde{\alpha}_{N}(s, \mathbf{x}^{(k-1)}, \underbrace{x_{k}, \cdots, x_{k}}_{N-k+1}, x) \triangleq \tilde{\alpha}_{N, k}\left(s, \mathbf{x}^{(k)}, x\right), \quad \text { if } s \in\left[t_{k-1}, t_{k}\right) ; \\
\tilde{\beta}_{N, k}\left(s, \mathbf{x}^{(k)}, x\right) \triangleq \sum_{i=1}^{k} \tilde{u}_{N x_{i}}\left(s, \mathbf{x}^{(k)}, x\right), \text { for } s \in\left[t_{k-1}, t_{k}\right) ; \\
\tilde{\beta}_{N}\left(s, \mathbf{x}^{(N)}, x\right) \equiv \tilde{\beta}_{N}(s, \mathbf{x}^{(k-1)}, \underbrace{x_{k}, \cdots, x_{k}}_{N-k+1}, x) \triangleq \tilde{\beta}_{N, k}\left(s, \mathbf{x}^{(k)}, x\right), \quad \text { if } s \in\left[t_{k-1}, t_{k}\right) .
\end{array}\right.
$$

Again by the Itô-Krylov formula,

$$
\begin{aligned}
\mathrm{d} & Y_{N, s}^{t, x}=-\tilde{f}_{N}\left(\Theta_{N, s}^{t}, \tilde{u}_{N}\left(\Theta_{N, s}^{t}\right), Z_{N, s}^{t, x}\right) \mathrm{d} s+Z_{N, s}^{t, x} \mathrm{~d} W(s)=\mathrm{d} \tilde{u}_{N}\left(\Theta_{N, s}^{t}\right) \\
= & \left\{\tilde{u}_{N s}\left(\Theta_{N, s}^{t}\right)+\tilde{u}_{N x}\left(\Theta_{N, s}^{t}\right) \tilde{b}_{N}\left(\Theta_{N, s}^{t}, Y_{N, s}^{t, x}\right)+\frac{1}{2} \sum_{i=1}^{k} \tilde{u}_{N x_{i} x_{i}}\left(\Theta_{N, s}^{t}\right)\right. \\
& \left.+\sum_{i=1}^{k} \tilde{u}_{N x_{i} x}\left(\Theta_{N, s}^{t}\right) \tilde{\sigma}_{N}\left(\Theta_{N, s}^{t}, Y_{N, s}^{t, x}\right)+\frac{1}{2} \tilde{u}_{N x x}\left(\Theta_{N, s}^{t}\right) \tilde{\sigma}_{N}^{2}\left(\Theta_{N, s}^{t}, Y_{N, s}^{t, x}\right)\right\} \mathrm{d} s \\
& +\left\{\sum_{i=1}^{k} \tilde{u}_{N x_{i}}\left(\Theta_{N, s}^{t}\right)+\tilde{u}_{N x}\left(\Theta_{N, s}^{t}\right) \tilde{\sigma}_{N}\left(\Theta_{N, s}^{t}, Y_{N, s}^{t, x}\right)\right\} \mathrm{d} W(s) \\
= & \left\{\tilde{\alpha}_{N}\left(\Theta_{N, s}^{t}\right)+\tilde{u}_{N x}\left(\Theta_{N, s}^{t}\right) \tilde{b}_{N}\left(\Theta_{N, s}^{t}, Y_{N, s}^{t, x}\right)+\tilde{\beta}_{N x}\left(\Theta_{N, s}^{t}\right) \tilde{\sigma}_{N}\left(\Theta_{N, s}^{t}, Y_{N, s}^{t, x}\right)\right. \\
& \left.+\frac{1}{2} \tilde{u}_{N x x}\left(\Theta_{N, s}^{t}\right) \tilde{\sigma}_{N}^{2}\left(\Theta_{N, s}^{t}, Y_{N, s}^{t, x}\right)\right\} \mathrm{d} s \\
& +\left\{\tilde{\beta}_{N}\left(\Theta_{N, s}^{t}\right)+\tilde{u}_{N x}\left(\Theta_{N, s}^{t}\right) \tilde{\sigma}_{N}\left(\Theta_{N, s}^{t}, Y_{N, s}^{t, x}\right)\right\} \mathrm{d} W(s) .
\end{aligned}
$$

Hence

$$
\begin{aligned}
\tilde{\alpha}_{N}\left(\Theta_{N, s}^{t}\right)= & -\tilde{f}_{N}\left(\Theta_{N, s}^{t} \tilde{u}_{N}\left(\Theta_{N, s}^{t}\right), Z_{N, s}^{t, x}\right)-\tilde{u}_{N x}\left(\Theta_{N, s}^{t}\right) \tilde{b}_{N}\left(\Theta_{N, s}^{t}, Y_{N, s}^{t, x}\right) \\
& -\tilde{\beta}_{N x}\left(\Theta_{N, s}^{t}\right) \tilde{\sigma}_{N}\left(\Theta_{N, s}^{t}, Y_{N, s}^{t, x}\right)-\frac{1}{2} \tilde{u}_{N x x}\left(\Theta_{N, s}^{t}\right) \tilde{\sigma}_{N}^{2}\left(\Theta_{N, s}^{t}, Y_{N, s}^{t, x}\right) .
\end{aligned}
$$

Note that $X_{N, s}^{s, x}=x, \Theta_{N, s}^{s}=\theta_{s}$ and $Y_{N, s}^{s, x}=\tilde{u}_{N}\left(\theta_{s}\right)$. Thus (25) and (28) imply

$$
\begin{aligned}
\mathrm{d} \tilde{u}_{N}\left(\theta_{s}\right) \\
=-\left\{\frac{1}{2} \tilde{u}_{N x x}\left(\theta_{s}\right) \tilde{\sigma}_{N}^{2}\left(\theta_{s}, \tilde{u}_{N}\right)+\tilde{u}_{N x}\left(\theta_{s}\right) \tilde{b}_{N}\left(\theta_{s}, \tilde{u}_{N}\right)+\tilde{\beta}_{N x}\left(\theta_{s}\right) \tilde{\sigma}_{N}\left(\theta_{s}, \tilde{u}_{N}\right)\right. \\
\left.+\tilde{f}_{N}\left(\theta_{s}, \tilde{u}_{N}\left(\theta_{s}\right), \tilde{\beta}_{N}\left(\theta_{s}\right)+\tilde{u}_{N x}\left(\theta_{s}\right) \tilde{\sigma}_{N}\left(\theta_{s}, \tilde{u}_{N}\right)\right)\right\} \mathrm{d} s+\tilde{\beta}_{N}\left(\theta_{s}\right) \mathrm{d} W(s) .
\end{aligned}
$$

Denote

$$
u_{N}(s, \omega, x) \triangleq \tilde{u}_{N}\left(\theta_{s}\right), \alpha_{N}(s, \omega, x) \triangleq \tilde{\alpha}_{N}\left(\theta_{s}\right) \text { and } \beta_{N}(s, \omega, x) \triangleq \tilde{\beta}_{N}\left(\theta_{s}\right) .
$$

Suppressing the variables $\left(s, \omega, x, \tilde{u}_{N}\right)$, one has

$$
\left\{\begin{array}{l}
\mathrm{d} u_{N}=-\left\{\frac{1}{2} u_{N x x} \sigma_{N}^{2}+\beta_{N x} \sigma_{N}+u_{N x} b_{N}+f_{N}\right\} \mathrm{d} s+\beta_{N} \mathrm{~d} W(s) ; \\
u_{N}(T, \omega, x)=g_{N}(\omega, x)
\end{array}\right.
$$

and $\left(u_{N}, \beta_{N}\right)$ is a weak solution to BSPDE (30). By the regularity argument in [3], we know that $u_{N}$ are uniformly bounded in supremum norm on every 
compact subset of $[0, T] \times \Omega \times \mathbb{R}$, and $u_{N x}$ are uniformly bounded in $\mathscr{H}^{0}$. Thus we can extract a subsequence (still indexed by $N$ ) such that it converges to some $u \in \mathscr{C}^{0}$. By Proposition 3.2, $u \in \mathscr{H}^{1}$ and there exists $\beta$ such that $(u, \beta)$ is a weak solution to BSPDE (7) with coefficients $(b, \sigma, f, g)$.

Step 2 (Weak Existence of the FBSDE): Now we use the decoupling field $u$ to decouple FBSDE (2). Let us consider the following forward SDE:

$$
X_{s}^{t, x}=x+\int_{t}^{s} b\left(r, X_{r}^{t, x}, u\left(r, X_{r}^{t, x}\right)\right) \mathrm{d} r+\int_{t}^{s} \sigma\left(r, X_{r}^{t, x}, u\left(r, X_{r}^{t, x}\right)\right) \mathrm{d} W_{r} .
$$

Without loss of generality in what follows we assume $t=0$. We claim that this SDE possesses a weak solution. Indeed, on any given probability space $(\Omega, \mathcal{F}, \mathbb{P})$ on which is defined a standard Brownian motion $W$, consider the following SDE:

$$
X_{t}=x+\int_{0}^{t} \sigma\left(r, X_{r}\right) \mathrm{d} W_{r} .
$$

Note that the function $u \in W_{2}^{1,2}([0, T] \times \mathbb{R})$ actually has a bounded spatial derivative $u_{x}$. Combining with (A.1), it is readily seen that the coefficient $\sigma(t, x)$ is uniformly Lipschitz in $x$. Thus the SDE (32) admits a unique strong solution, denoted by $X=X^{0, x}$.

Next, define $\theta=\frac{b}{\sigma}$, which is bounded, thanks to (A.1) and (A.2). Thus

$$
M_{t} \triangleq \exp \left\{\int_{0}^{t} \theta\left(s, X_{s}\right) \mathrm{d} W_{s}-\frac{1}{2} \int_{0}^{t}\left|\theta\left(s, X_{s}\right)\right|^{2} \mathrm{~d} s\right\}, \quad t \geq 0
$$

is a martingale under $\mathbb{P}$. Now define $\left.\frac{\mathrm{d} \mathbb{Q}}{\mathrm{d} \mathbb{P}}\right|_{\mathcal{F}_{t}}=M_{t}, t \in[0, T]$. Then by the Girsanov theorem ([16], Theorem 8.9.4), under $\mathbb{Q}$ the process $\bar{W}_{t}=W_{t}-\int_{0}^{t} \theta\left(s, X_{s}^{0, x}\right) \mathrm{d} s$ is a Brownian motion, and $X$ satisfies (31). Let us abuse the notation and denote $\bar{W}$ by $W$ again. In other words, we have shown that there exist a process $X_{s}^{t, x}$ and Wiener process, still denoted by $W$, such that $\left(\Omega, \mathcal{F}, \mathbb{P} ; W, X_{s}^{t, x}\right)$ is a weak solution of (31). Let us define

$$
\left\{\begin{array}{l}
Y_{s}^{t, x} \triangleq u\left(s, X_{s}^{t, x}\right) \\
Z_{s}^{t, x} \triangleq \beta\left(s, X_{s}^{t, x}\right)+u_{x}\left(s, X_{s}^{t, x}\right) \sigma\left(s, X_{s}^{t, x}, Y_{s}^{t, x}\right) .
\end{array}\right.
$$

We would like to show that $\left(\Omega, \mathcal{F}, \mathbb{P} ; W, X_{s}^{t, x}, Y_{s}^{t, x}, Z_{s}^{t, x}\right)$ is a weak solution of FBSDE (2). Since $\tilde{u}_{N}$ are bounded in $W_{2}^{1,2}$, uniformly over $N$, we know $u \in W_{2}^{1,2}$ for $\mathbb{P}$-a.s. $\omega$. By the construction of $\tilde{\alpha}_{N}$ and $\tilde{\beta}_{N}$, it is also clear that $\alpha \in \mathscr{H}^{0}$ and $\beta \in \mathscr{C}^{1}$, where $\alpha$ is defined in (8). Thus by Lemma 4.1, one has

$$
\begin{aligned}
& \mathrm{d} Y_{s}^{t, x}=\mathrm{d} u\left(s, X_{s}^{t, x}\right) \\
& =\left\{\alpha\left(s, X_{s}^{t, x}\right)+u_{x}\left(s, X_{s}^{t, x}\right) b\left(s, X_{s}^{t, x}, Y_{s}^{t, x}\right)+\frac{1}{2} u_{x x}\left(s, X_{s}^{t, x}\right) \sigma^{2}\left(s, X_{s}^{t, x}, Y_{s}^{t, x}\right)\right. \\
& \left.+\beta_{x}\left(s, X_{s}^{t, x}\right) \sigma\left(s, X_{s}^{t, x}, Y_{s}^{t, x}\right)\right\} \mathrm{d} s+Z_{s}^{t, x} \mathrm{~d} W_{s} \\
& =f\left(s, X_{s}^{t, x}, Y_{s}^{t, x}, Z_{s}^{t, x}\right) \mathrm{d} s+Z_{s}^{t, x} \mathrm{~d} W_{s},
\end{aligned}
$$


which completes the proof of Step 2.

Step 3 (The General Case): Now let us consider the general case, that is, we proceed without requiring the smoothness of $\tilde{b}_{N}, \tilde{\sigma}_{N}, \tilde{f}_{N}$ and $\tilde{g}_{N}$. For each $N$ let us consider the standard smooth mollifiers $\left\{\tilde{b}_{N}^{(l)}, \tilde{\sigma}_{N}^{(l)}, \tilde{f}_{N}^{(l)}, \tilde{g}_{N}^{(l)}\right\}_{l=1}^{\infty}$. Based on standard BSPDE result, it is easy to check that the conditions 1) - 4) in Proposition 3.2 hold. Following closely to Step 1 and Step 2, utilizing the stability result from Proposition 3.2, we conclude that FBSDE (2) admits a weak solution.

\section{Weak Uniqueness}

Now we are ready to prove the main result of this paper.

Theorem 5.1 Under Assumption 2.1, the FBSDE (2) admits a unique weak solution.

Proof. The weak existence has been shown in Proposition 4.2. Now suppose there is another weak solution $\left(\hat{\Omega}, \hat{\mathcal{F}}, \hat{\mathbb{P}}, \hat{W}, \hat{X}_{s}^{t, x}, \hat{Y}_{s}^{t, x}, \hat{Z}_{s}^{t, x}\right)$ of (2). Applying the general Itô-Ventzell formula to get

$$
\begin{aligned}
d & \left\{u\left(s, \hat{X}_{s}^{t, x}\right)-\hat{Y}_{s}^{t, x}\right\} \\
= & \left\{u_{t}\left(s, \hat{X}_{s}^{t, x}\right)+\frac{1}{2} u_{x x}\left(s, \hat{X}_{s}^{t, x}\right) \sigma^{2}\left(s, \hat{X}_{s}^{t, x}, u\left(s, \hat{X}_{s}^{t, x}\right)\right)\right. \\
& \left.+u_{x}\left(s, \hat{X}_{s}^{t, x}\right) b\left(s, \hat{X}_{s}^{t, x}, u\left(s, \hat{X}_{s}^{t, x}\right)\right)\right\} \mathrm{d} s \\
& +u_{x}\left(s, \hat{X}_{s}^{t, x}\right) \sigma\left(s, \hat{X}_{s}^{t, x}, u\left(s, \hat{X}_{s}^{t, x}\right)\right) \mathrm{d} \hat{W}_{s} \\
& -h\left(s, \hat{X}_{s}^{t, x}, \hat{Y}_{s}^{t, x}\right) \mathrm{d} s-\hat{Z}_{s}^{t, x} \mathrm{~d} \hat{W}_{s} \\
= & \left\{h\left(s, \hat{X}_{s}^{t, x}, u\left(s, \hat{X}_{s}^{t, x}\right)\right)-h\left(s, \hat{X}_{s}^{t, x}, \hat{Y}_{s}^{t, x}\right)\right\} \mathrm{d} s \\
& +\left\{u_{x}\left(s, \hat{X}_{s}^{t, x}\right) \sigma\left(s, \hat{X}_{s}^{t, x}, u\left(s, \hat{X}_{s}^{t, x}\right)\right)-\hat{Z}_{s}^{t, x}\right\} \mathrm{d} \hat{W}_{s} .
\end{aligned}
$$

Note that $h$ is Lipschitz continuous with Lipschitz constant $K$. Thus by Itô-Krylov formula again, one has

$$
\begin{aligned}
& E\left|u\left(s, \hat{X}_{s}^{t, x}\right)-\hat{Y}_{s}^{t, x}\right|^{2}+E \int_{s}^{T}\left|u_{x}\left(r, \hat{X}_{r}^{t, x}\right) \sigma\left(r, \hat{X}_{r}^{t, x}, u\left(r, \hat{X}_{r}^{t, x}\right)\right)-\hat{Z}_{r}^{t, x}\right|^{2} \mathrm{~d} r \\
& =-E \int_{s}^{T} 2\left(u\left(r, \hat{X}_{r}^{t, x}\right)-\hat{Y}_{r}^{t, x}\right)\left(h\left(r, \hat{X}_{r}^{t, x}, u\left(r, \hat{X}_{r}^{t, x}\right)\right)-h\left(r, \hat{X}_{r}^{t, x}, \hat{Y}_{r}^{t, x}\right)\right) \mathrm{d} r \\
& \leq 2 K E \int_{s}^{T}\left|u\left(r, \hat{X}_{r}^{t, x}\right)-\hat{Y}_{r}^{t, x}\right|^{2} \mathrm{~d} r .
\end{aligned}
$$

An application of the Gronwall inequality implies

$$
u\left(s, \hat{X}_{s}^{t, x}\right)=\hat{Y}_{s}^{t, x} \text { and } \hat{Z}_{s}^{t, x}=u_{x}\left(s, \hat{X}_{s}^{t, x}\right) \sigma\left(s, \hat{X}_{s}^{t, x}, u\left(s, \hat{X}_{s}^{t, x}\right)\right), \hat{P} \text {-a.s. }
$$

Thus we shall consider the following decoupled forward system:

$$
\bar{X}_{s}^{t, x}=x+\int_{t}^{s} b\left(r, \bar{X}_{r}^{t, x}, u\left(r, \bar{X}_{r}^{t, x}\right)\right) \mathrm{d} r+\int_{t}^{s} \sigma\left(r, \bar{X}_{r}^{t, x}, u\left(r, \bar{X}_{r}^{t, x}\right)\right) \mathrm{d} W_{r} .
$$

Step 3 (Forward Weak Uniqueness): From previous steps, clearly $\left(X_{s}^{t, x} ; \Omega, \mathcal{F}, P, W\right)$ and $\left(\hat{X}_{s}^{t, x} ; \hat{\Omega}, \hat{\mathcal{F}}, \hat{P}, \hat{W}\right)$ are both weak solutions to (35).

Let $\bar{b}(s, x) \triangleq b(s, x, u(s, x)), \quad \bar{\sigma}(s, x) \triangleq \sigma(s, x, u(s, x))$, and 
$\theta(s, x) \triangleq \frac{\bar{b}(s, x)}{\bar{\sigma}(s, x)}$ (note that the system is strictly non-degenerate). It is easy to see that

$$
M_{s} \triangleq \exp \left\{-\int_{t}^{s} \theta\left(r, X_{r}^{t, x}\right) \mathrm{d} W_{r}-\frac{1}{2} \int_{t}^{s}\left|\theta\left(r, X_{r}^{t, x}\right)\right|^{2} \mathrm{~d} r\right\}
$$

and

$$
\hat{M}_{s} \triangleq \exp \left\{-\int_{t}^{s} \theta\left(r, \hat{X}_{r}^{t, x}\right) \mathrm{d} \hat{W}_{r}-\frac{1}{2} \int_{t}^{s}\left|\theta\left(r, \hat{X}_{r}^{t, x}\right)\right|^{2} \mathrm{~d} r\right\}
$$

are martingales under probabilities $P$ and $\hat{P}$, respectively. Define new probabilities $P_{1}$ and $P_{2}$ by

$$
\frac{\mathrm{d} P_{1}}{\mathrm{~d} P}=M_{T} \text { and } \frac{\mathrm{d} P_{2}}{\mathrm{~d} \hat{P}}=\hat{M}_{T}
$$

By the Girsanov theorem, $W_{s}^{1} \triangleq W_{s}+\int_{t}^{s} \theta\left(r, X_{r}^{t, x}\right) \mathrm{d} r$ and $W_{s}^{2} \triangleq \hat{W}_{s}+\int_{t}^{s} \theta\left(r, \hat{X}_{r}^{t, x}\right) \mathrm{d} r$ are Brownian motions under $\left(\Omega, \mathcal{F}, P_{1}\right)$ and $\left(\hat{\Omega}, \hat{\mathcal{F}}, P_{2}\right)$, respectively. Since strong uniqueness holds for

$$
\bar{X}_{s}^{t, x}=x+\int_{t}^{s} \sigma\left(r, \bar{X}_{r}^{t, x}, u\left(r, \bar{X}_{r}^{t, x}\right)\right) \mathrm{d} W_{r},
$$

and $\left(X_{s}^{t, x} ; \Omega, \mathcal{F}, P_{1}, W^{1}\right)$ and $\left(\hat{X}_{s}^{t, x} ; \hat{\Omega}, \hat{\mathcal{F}}, P_{2}, W^{2}\right)$ are both weak solutions to the above equation, we know that the distribution of $\left(X_{s}^{t, x}, W_{s}^{1}\right)$ under $P_{1}$ coincides with the distribution of $\left(\hat{X}_{s}^{t, x}, W_{s}^{2}\right)$ under $P_{2}$, i.e., for any bounded measurable functional $f: C([0, T]) \times C([0, T]) \rightarrow \mathbb{R}$,

$$
E^{P_{1}}\left[f\left(X_{.}^{t, x}, W^{1}\right)\right]=E^{P_{2}}\left[f\left(\hat{X}_{\cdot}^{t, x}, W^{2}\right)\right] .
$$

Now for any $f: C([0, T]) \rightarrow \mathbb{R}$, by (36),

$$
\begin{aligned}
& E^{P}\left[f\left(X^{t, x}\right)\right]=\int_{\Omega} f\left(X^{t, x}\right) \frac{\mathrm{d} P}{\mathrm{~d} P_{1}} \mathrm{~d} P_{1}=E^{P_{1}}\left[f\left(X^{t, x}\right) \frac{1}{M_{T}}\right] \\
& =E^{P_{1}}\left[f\left(X^{t, x}\right) \exp \left\{\int_{t}^{T} \theta\left(r, X_{r}^{t, x}\right) \mathrm{d} W_{r}+\frac{1}{2} \int_{t}^{T}\left|\theta\left(r, X_{r}^{t, x}\right)\right|^{2} \mathrm{~d} r\right\}\right] \\
& =E^{P_{1}}\left[f\left(X^{t, x}\right) \exp \left\{\int_{t}^{T} \theta\left(r, X_{r}^{t, x}\right) \mathrm{d} W_{r}^{1}-\frac{1}{2} \int_{t}^{T}\left|\theta\left(r, X_{r}^{t, x}\right)\right|^{2} \mathrm{~d} r\right\}\right] \\
& =E^{P_{2}}\left[f\left(\hat{X}^{t, x}\right) \exp \left\{\int_{t}^{T} \theta\left(r, \hat{X}_{r}^{t, x}\right) \mathrm{d} W_{r}^{2}-\frac{1}{2} \int_{t}^{T}\left|\theta\left(r, \hat{X}_{r}^{t, x}\right)\right|^{2} \mathrm{~d} r\right\}\right] \\
& =E^{P_{2}}\left[f\left(\hat{X}^{t, x}\right) \exp \left\{\int_{t}^{T} \theta\left(r, \hat{X}_{r}^{t, x}\right) \mathrm{d} \hat{W}_{r}+\frac{1}{2} \int_{t}^{T}\left|\theta\left(r, \hat{X}_{r}^{t, x}\right)\right|^{2} \mathrm{~d} r\right\}\right] \\
& =E^{P_{2}}\left[f\left(\hat{X}^{t, x}\right) \frac{1}{\hat{M}_{T}}\right]=\int_{\Omega} f\left(\hat{X}^{t, x}\right) \frac{\mathrm{d} \hat{P}}{\mathrm{~d} P_{2}} \mathrm{~d} P_{2}=E^{\hat{P}}\left[f\left(\hat{X}^{t, x}\right)\right] .
\end{aligned}
$$

Thus we have shown that the distribution of $X_{s}^{t, x}$ under $P$ coincides with the distribution of $\hat{X}_{s}^{t, x}$ under $\hat{P}$. By [17], we know that the distribution of $\left(X_{s}^{t, x}, W_{s}\right)$ under $P$ coincides with the distribution of $\left(\hat{X}_{s}^{t, x}, \hat{W}_{s}\right)$ under $\hat{P}$.

Step 4 (Backward Weak Uniqueness): We know $u$ is continuous, hence Le- 
besgue measurable. Note that

$$
\begin{gathered}
Y_{s}^{t, x}=u\left(s, X_{s}^{t, x}\right), \\
Z_{s}^{t, x}=\sigma\left(s, X_{s}^{t, x}, u\left(s, X_{s}^{t, x}\right)\right) u_{x}\left(s, X_{s}^{t, x}\right) .
\end{gathered}
$$

Hence it is easy to conclude that the law of $\left(X_{s}^{t, x}, Y_{s}^{t, x}, Z_{s}^{t, x}, W_{s}\right)$ under $P$ coincides with the law of $\left(\hat{X}_{s}^{t, x}, \hat{Y}_{s}^{t, x}, \hat{Z}_{s}^{t, x}, \hat{W}_{s}\right)$ under $\hat{P}$.

\section{Conclusion}

The well-posedness in weak sense of a type of fully coupled FBSDE has been established. The difficulty of the problem stems from the fact that the forward drift coefficient may be discontinuous. The existence and uniqueness of a strong solution for such FBSDE remain to be an open problem. The author plans to attack this issue in his future research.

\section{Conflicts of Interest}

The author declares no conflicts of interest regarding the publication of this paper.

\section{References}

[1] Antonelli, F. and Ma, J. (2003) Weak Solutions of Forward-Backward SDE's. Stochastic Analysis and Applications, 21, 493-514. https://doi.org/10.1081/SAP-120020423

[2] Ma, J., Zhang, J. and Zheng, Z. (2008) Weak Solutions for Backward Stochastic Differential Equations, a Martingale Approach. The Annals of Probability, 36, 2092-2125. https://doi.org/10.1214/08-AOP0383

[3] Chen, J., Ma, J. and Yin, H. (2018) Forward-Backward Stochastic Differential Equations with Discontinuous Coefficients. Stochastic Analysis and Applications, 36, 274-294. https://doi.org/10.1080/07362994.2017.1399799

[4] Bali, T. (2003) Modeling the Stochastic Behavior of Short-Term Interest Rates: Pricing Implications for Discount Bonds. Journal of Banking and Finance, 27, 201-228. https://doi.org/10.1016/S0378-4266(01)00216-3

[5] Driffill, J., Kenc, T. and Sola, M. (2003) An Empirical Examination of Term Structure Models with Regime Shifts. EFMA 2003 Helsinki Meetings. https://doi.org/10.2139/ssrn.393481

[6] Bansal, R. and Zhou, H. (2002) Term Structure of Interest Rates with Regime Shifts. Journal of Finance, 57, 1997-2043. https://doi.org/10.1111/0022-1082.00487

[7] Dai, Q., Singleton, K. and Yang, W. (2007) Regime Shifts in a Dynamic Term Structure Model of U.S. Treasury Bond Yields. Review of Financial Studies, 20, 1669-1706. https://doi.org/10.1093/rfs/hhm021

[8] Evans, M. (2003) Real Risk, Inflation Risk, and the Term Structure. The Economic Journal, 113, 345-389. https://doi.org/10.1111/1468-0297.00130

[9] Landen, C. (2000) Bond Pricing in a Hidden Markov Model of the Short Rate. Finance and Stochastics, 4, 371-389. https://doi.org/10.1007/PL00013526

[10] Duffie, D., Ma, J. and Yong, J. (1995) Black's Console Rate Conjecture. The Annals of Applied Probability, 5, 356-382. https://doi.org/10.1214/aoap/1177004768 
[11] Ma, J., Wu, Z., Zhang, D. and Zhang, J. (2015) On Well-Posedness of Forward-Backward SDES: A Unified Approach. The Annals of Applied Probability, 25, 2168-2214. https://doi.org/10.1214/14-AAP1046

[12] Ma, J., Yin, H. and Zhang, J. (2014) On Non-Markovian Forward-Backward SDEs and Backward Stochastic PDEs. Stochastic Processes and Their Applications, 122, 3980-4004. https://doi.org/10.1016/j.spa.2012.08.002

[13] Pardoux, E. (1979) Stochastic Partial Differential Equations and Filtering of Diffusion Processes. Stochastics, 3, 127-167. https://doi.org/10.1080/17442507908833142

[14] Krylov, N.V. (1980) Controlled Diffusion Processes. Springer-Verlag, Berlin. https://doi.org/10.1007/978-1-4612-6051-6

[15] Kim, D. and Krylov, N.V. (2007) Parabolic Equations with Measurable Coefficients. Potential Analysis, 26, 345-361. https://doi.org/10.1007/s11118-007-9042-8

[16] Kuo, H.-H. (2000) Introduction to Stochastic Integration. Springer, Berlin.

[17] Brossard, J. (2003) Deux notions équivalentes d'unicité en loi pour les équations différentielles stochastiques. In: Séminaire de Probabilités XXXVII, Volume 1832, Springer, Berlin, 246-250. https://doi.org/10.1007/978-3-540-40004-2_10 